\title{
Effects of Plastic Anisotropy and Void Shape on Full Three-Dimensional Void Growth
}

\section{Legarth, Brian Nyvang; Tvergaard, Viggo}

Published in:

Journal of Applied Mechanics

Link to article, DOI:

$10.1115 / 1.4039172$

Publication date:

2018

Document Version

Peer reviewed version

Link back to DTU Orbit

Citation (APA):

Legarth, B. N., \& Tvergaard, V. (2018). Effects of Plastic Anisotropy and Void Shape on Full Three-Dimensional Void Growth. Journal of Applied Mechanics, 85(5), [051007]. https://doi.org/10.1115/1.4039172

\section{General rights}

Copyright and moral rights for the publications made accessible in the public portal are retained by the authors and/or other copyright owners and it is a condition of accessing publications that users recognise and abide by the legal requirements associated with these rights.

- Users may download and print one copy of any publication from the public portal for the purpose of private study or research.

- You may not further distribute the material or use it for any profit-making activity or commercial gain

- You may freely distribute the URL identifying the publication in the public portal

If you believe that this document breaches copyright please contact us providing details, and we will remove access to the work immediately and investigate your claim 


\section{Effects of Plastic Anisotropy and Void Shape on Full Three-Dimensional Void Growth}

\author{
Brian Nyvang Legarth \\ Associate professor \\ Department of Mechanical Engineering \\ Solid Mechanics \\ Technical university of Denmark \\ DK-2800 Kgs. Lyngby, Denmark \\ Email: bnl@mek.dtu.dk
}

\author{
Viggo Tvergaard \\ Professor Emeritus \\ Department of Mechanical Engineering \\ Solid Mechanics \\ Technical university of Denmark \\ DK-2800 Kgs. Lyngby, Denmark \\ Email: viggo@mek.dtu.dk
}

Void growth in an anisotropic ductile solid is studied by numerical analyses for three dimensional unit cells initially containing a void. The effect of plastic anisotropy on void growth is the main focus, but the studies include effects of different void shapes, including oblate, prolate or general ellipsoidal voids. Also other $3 D$ effects such as those of different spacings of voids in different material directions, and effects of different macroscopic principal stresses in three directions are accounted for. It is found that the presence of plastic anisotropy amplifies the differences between predictions obtained for different initial void shapes. Also, differences between principal transverse stresses show a strong interaction with the plastic anisotropy, such that the response is very different for different anisotropies. The studies are carried out for one particular choice of void volume fraction and stress triaxiality.

\section{Introduction}

Void growth in ductile metals, leading to ductile fracture under tensile stresses, has been modelled early on by McClintock [1] and Rice and Tracey [2], and subsequently a large amount of research has focussed on this area (see reviews by Garrison and Moody [3]; Tvergaard [4]; Benzerga and Leblond [5]; Benzerga et al. [6]). Most models for ductile porous materials have considered initially spherical voids, uniformly distributed in a standard elastic-plastic material with isotropic hardening. A much used set of constitutive equations for porous ductile solids have been developed by Gurson [7] and extended by Tvergaard [8] and Tvergaard and Needleman [9].

Plastic anisotropy is often present in components due to processing such as rolling, extrusion or forging. When the resistance to plastic flow is different for different directions in the material, this will also affect void growth and the final occurrence of ductile fracture. Often, plastic anisotropy has been represented by using the anisotropic yield surface proposed by Hill [10,11]. Benzerga and Besson [12] appear to be the first to consider void growth in a Hill matrix. They used axisymmetric cell models for initially spherical voids to develop an extension of the Gurson model. Subsequently, Keralavarma and Benzerga [13] extended this, considering initially oblate or prolate voids, and Morin et al. [14] used a new limit analysis for Hill materials for various spheroidal cells containing a confocal spheroidal void, to calculate approximate yield surfaces.

Full three-dimensional unit cell calculations have been carried out by Steglich et al. [15], using a cube cell with an initially spherical void to study differences between tension in three different directions with respect to the rolling direction of a sheet. Also Chien et al. [16] and Wang et al. [17] used a unit cell in the form of a cube with a spherical void growth in a Hill material. In Dæhli et al. [18] the anisotropic material is described by the phenomenological yield criterion proposed by Barlat et al. [19], with this material model calibrated by a total number of 500 distinct yield points, obtained by using numerical simulations with the full-constraint Taylor method. Also the studies in [18] use a cube unit cell with an initially spherical void located at the centre. Somewhat related to these analyses are also the numerical studies for creep in a porous FCC single crystal by Srivastava et al. [20], where periodic boundary conditions have been used for the 3D unit cell. The authors [21] have earlier analysed the effect of 3D stress states on cavitation instabilities in an anisotropic solid, using a full transient analysis of the equations of motion to approximate the quasi-static solution.

The aim of the present paper is to study the effect of anisotropic plasticity on void growth, taking into account the influence of void shape, the influence of different 3D stress states, and the influence of different spacings between voids in the periodic patterns analysed. The voids are taken to be initially spheroidal, including oblate, prolate and general ellipsoidal shapes with different axes in all three principal directions. The plastic anisotropy is here described by Hill's quadratic yield criterion.

\section{Problem Formulation and Material Model}

A periodic distribution of voids in a plastically anisotropic metal is analyzed by a unit cell approach. Fig. 1 shows the geometry with the dimensions and coordinate axes used. The reference Cartesian coordinate system is denoted by $x_{i}$. As shown in Fig. $1 x_{i}$ is aligned with the sides of the 


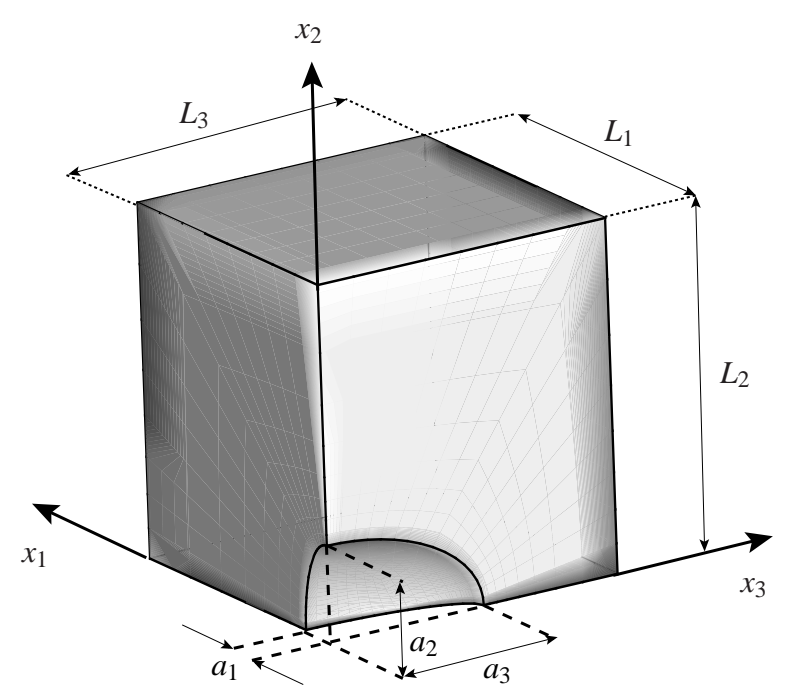

Fig. 1. Geometry of ellipsoidal void analyzed. Illustrated for $w_{1}=$ 2 and $w_{3}=\frac{1}{2}$ as well as $L_{2} / L_{1}=L_{2} / L_{3}=1$ for an initial void volume fraction of $f_{0}=0.01$.

domain analyzed. Also the principal axes of anisotropy, $\hat{x}_{i}$, are initially aligned with the sides in every material point (not shown). However, as no shear load is applied on the sides of the unit cell, the axes do not rotate here, and then the symmetries of the problem are maintained throughout the load history. Hence, even for a plastically anisotropic material, a macroscopic shear strain will not evolve if a pure normal stress state is introduced macroscopically. Exploiting this, only one-eight of the unit cell needs to be analyzed. The general 3D ellipsoidal void shape defined by the three initial half axes, $a_{1}, a_{2}$ and $a_{3}$, with the initial half spacings $L_{1}, L_{2}$ and $L_{3}$, then yields the initial void volume fraction, $f_{0}$, as

$$
f_{0}=\left(\frac{\pi a_{1} a_{2} a_{3}}{6 L_{1} L_{2} L_{3}}\right)_{0}
$$

where the initial aspect ratios for the void shape are $w_{1}=$ $a_{2} / a_{1}$ and $w_{3}=a_{2} / a_{3}$.

Denoting the time derivative by a superposed dot, i.e. $\frac{\partial(\bullet)}{\partial t}=(\bullet)$, the boundary conditions are expressed in terms of the displacement rates, $\dot{u}_{i}$, and the surface tractions, $\dot{T}_{i}$, as

$$
\begin{array}{lllll}
\dot{u}_{1}=0 & ; & \dot{T}_{2}=\dot{T}_{3}=0 & \text { at } & x_{1}=0 \\
\dot{u}_{2}=0 & ; & \dot{T}_{1}=\dot{T}_{3}=0 & \text { at } & x_{2}=0 \\
\dot{u}_{3}=0 & ; & \dot{T}_{1}=\dot{T}_{2}=0 & \text { at } & x_{3}=0 \\
\dot{u}_{1}=\Delta_{1} & ; & \dot{T}_{2}=\dot{T}_{3}=0 & \text { at } & x_{1}=L_{1} \\
\dot{u}_{2}=\Delta_{2} & ; & \dot{T}_{1}=\dot{T}_{3}=0 & \text { at } & x_{2}=L_{2} \\
\dot{u}_{3}=\Delta_{3} & ; & \dot{T}_{1}=\dot{T}_{2}=0 & \text { at } & x_{3}=L_{3}
\end{array}
$$

where $\Delta_{1}, \Delta_{2}$ and $\Delta_{3}$ are prescribed displacement quantities used to introduce the load. The load is characterized by the three true normal stress components, $\Sigma_{1}, \Sigma_{2}$ and $\Sigma_{3}$, acting in the three coordinate directions, $x_{i}$. Taking $x_{2}$ as the primary loading direction, the stress components are related such that $\Sigma_{1}=\kappa_{1} \Sigma_{2}$ and $\Sigma_{3}=\kappa_{3} \Sigma_{2}$, where $\kappa_{1}$ and $\kappa_{3}$ are prescribed ratios. Two techniques have been used to enforce these stress ratios. The first method is an approximative method where initially $\Delta_{1}, \Delta_{2}$ and $\Delta_{3}$ are calculated as if the domain is purely elastic and without any void. Hereafter, the stresses are evaluated and $\Delta_{1}, \Delta_{2}$ and $\Delta_{3}$ are adjusted accordingly, which might take several increments in order to obtain the prescribed stress ratios. The method is straightforward to implement. In some analyses it has been necessary to use the combined finite element and Rayleigh-Ritz procedure as proposed by [22], which requires a set of trial-functions to be evaluated for each load increment. Each trial function represents the displacement of the nodes of one straight cell side that is being displaced. The method is exact, but is more demanding in terms of implementation. It is noted that both techniques yield the same results, but the latter seems to be numerically more stable.

The stress triaxiality, $T$, is

$$
T=\frac{\Sigma_{h}}{\Sigma_{e}}=\frac{\Sigma_{1}+\Sigma_{2}+\Sigma_{3}}{3 \Sigma_{e}}=\frac{\left(\kappa_{1}+1+\kappa_{3}\right) \Sigma_{2}}{3 \Sigma_{e}}
$$

with the effective stress taken as $\Sigma_{e}=\max \left(\left|\Sigma_{2}-\Sigma_{1}\right|, \mid \Sigma_{1}-\right.$ $\left.\Sigma_{3}|,| \Sigma_{2}-\Sigma_{3} \mid\right)$.

The elasto-plastically anisotropic material model used accounts for small elastic but finite plastic deformations in a visco-plastic formulation. The components of the secondorder velocity gradient tensor, $\mathbf{L}$, are determined by $L_{i j}=$ $\frac{\partial \dot{u}_{i}}{\partial x_{j}}=\dot{u}_{i, j}$, where the velocity field components for the material are $\dot{u}_{i}$. The symmetric part of $\mathbf{L}$ is the strain rate, $\mathbf{D}$, and the antisymmetric part is the continuum spin tensor, $\mathbf{W}$. The kinematics can then be written as [23-25]

$$
\mathbf{D}=\mathbf{D}^{\mathrm{e}}+\mathbf{D}^{\mathrm{p}} \quad ; \quad \mathbf{W}=\omega+\mathbf{W}^{\mathrm{p}} \quad ; \quad \mathbf{L}=\mathbf{D}+\mathbf{W}
$$

where the superscripts e and $\mathrm{p}$ denote the elastic and plastic parts, respectively, and $\omega$ is the spin of the substructure, whereas $\mathbf{W}^{\mathrm{p}}$ is the plastic spin, which will be neglected here, i.e. $\mathbf{W}^{\mathrm{p}}=\mathbf{0}$.

The elasticity relations are assumed to be given by Hooke's law. For the numerical implementation a symmetric stiffness matrix is pursued. Hence, the objective rate with respect to $\omega,\left(^{\nabla}\right)$, of the symmetric Kirchhoff stress, $\tau$, rather than the Cauchy stress, $\sigma$, is introduced as [30]

$$
\stackrel{\nabla}{\tau}=\mathbf{C}: \mathbf{D}^{\mathrm{e}}=\mathbf{C}:\left(\mathbf{D}-\mathbf{D}^{\mathrm{p}}\right)
$$

Here, $\mathbf{C}$ are the isotropic elastic moduli determined by Young's modulus, $E$, and Poisson's ratio, $v$. The plastic part of the strain rate is

$$
\mathbf{D}^{\mathrm{p}}=\dot{\phi} \mathbf{N}^{\mathrm{p}} \quad ; \quad \dot{\phi}=\dot{\varepsilon}_{0}\left(\frac{J}{g}\right)^{1 / m} \quad ; \quad \mathbf{N}^{\mathrm{p}}=\frac{\partial J}{\partial \sigma}
$$


Here, $J$ is value of the anisotropic yield function to be specified later and $\mathbf{N}^{\mathrm{p}}$ are the normals to the yield surface, which gives the direction of the plastic strain increment. The magnitude of the plastic strain increment is $\dot{\phi}, \dot{\varepsilon}_{0}$ is a reference strain rate, $m$ is a strain rate sensitivity parameter and $g=g\left(\varepsilon^{\mathrm{p}}\right)$ is an isotropic deformation dependent power-law hardening function

$$
g\left(\varepsilon^{\mathrm{p}}\right)=\sigma_{0}\left(1+\frac{\varepsilon^{\mathrm{p}}}{\varepsilon_{0}}\right)^{n}
$$

where $\sigma_{0}$ is the initial uniaxial yield stress in the $x_{1}$ direction, $\varepsilon_{0}=\sigma_{0} / E, n$ is the hardening exponent and the accumulated effective plastic strain, $\varepsilon^{\mathrm{p}}$, is

$$
\varepsilon^{\mathrm{p}}=\int \dot{\varepsilon}^{\mathrm{p}} \mathrm{d} t \quad ; \quad \dot{\varepsilon}^{\mathrm{p}}=\dot{\phi} \sqrt{\frac{2}{3} \mathbf{N}^{\mathrm{p}}: \mathbf{N}^{\mathrm{p}}}
$$

The classical quadratic yield function proposed by Hill $[10,11]$ is adopted here. Referring the stresses to the principal axes of anisotropy, $\hat{x}_{i}$, defined by the orthonormal basis, $\mathbf{n}_{i}$, such that $\hat{\sigma}_{i j}=\mathbf{n}_{i} \cdot \sigma \cdot \mathbf{n}_{i}$ for $(i, j)=1,2,3$, the anisotropic yield function is

$$
\begin{aligned}
J\left(\sigma, \mathbf{n}_{i}\right)= & \sqrt{\frac{3}{2(F+G+H)}}\left[F\left(\hat{\sigma}_{22}-\hat{\sigma}_{33}\right)^{2}+G\left(\hat{\sigma}_{33}-\hat{\sigma}_{11}\right)^{2}+\right. \\
& \left.H\left(\hat{\sigma}_{11}-\hat{\sigma}_{22}\right)^{2}+2 N \hat{\sigma}_{12}^{2}+2 L \hat{\sigma}_{23}^{2}+2 M \hat{\sigma}_{13}^{2}\right]^{1 / 2}
\end{aligned}
$$

The criterion is valid for six non-zero stress components and the coefficients of anisotropy, $F, G, H, N, L$ and $M$, can be determined by uniaxial tensile tests. A fairly simple model of evolving anisotropy (e.g. texture evolution) can be established by allowing the coefficients of anisotropy, $F, G, H, N, L$ and $M$, change with the deformations. Thus, during the deformation an initially isotropic material can evolve into an anisotropic one, or initial anisotropy can grow more pronounced [26]. However, here the coefficients of anisotropy are assumed to remain constant. During the deformation the orthonormal basis rotates according to $\dot{\mathbf{n}}_{i}=\omega \mathbf{n}_{i}$ as the principal axes of anisotropy are attached to the substructure of the material. This rotation will not destroy the symmetry of the problem, as the rotation is also symmetric around symmetry planes, such as the sides of the unit cell analyzed here.

The deformation history will be calculated in a linear incremental manner. In order to increase the stable time step, $\Delta t$, at any time, $t$, the rate tangent modulus method is used [27]. This is a forward gradient method based on an estimate of the plastic strain rate in the interval between $t$ and $t+$ $\Delta t$ defined by $\rho \in[0 ; 1]$. The final form of the constitutive relations can then be written as $[28,30]$

$$
\dot{\sigma}=\stackrel{\nabla}{\tau}+\omega \sigma+\sigma \omega-\sigma \operatorname{tr}(\mathbf{D})
$$

with

$$
\begin{aligned}
& \stackrel{\nabla}{\tau}=\tilde{\mathbf{C}}: \mathbf{D}-\mathbf{P} \\
& \tilde{\mathbf{C}}=\mathbf{C}-\frac{\xi}{h(1+\xi)}\left(\mathbf{C}: \mathbf{N}^{\mathrm{p}}\right) \otimes\left(\mathbf{N}^{\mathrm{p}}: \mathbf{C}\right) \\
& \mathbf{P}=\frac{\dot{\phi}_{t}}{1+\xi}\left(\mathbf{C}: \mathbf{N}^{\mathrm{p}}\right) \\
& \xi=\rho \Delta t\left(\frac{\partial \dot{\phi}}{\partial J}\right)_{t} h \quad ; \quad \rho \in[0 ; 1] \\
& h=\mathbf{N}^{\mathrm{p}}: \mathbf{C}: \mathbf{N}^{\mathrm{p}}-\left(\frac{\partial \dot{\phi}}{\partial \varepsilon^{\mathrm{p}}}\right)_{t}\left(\frac{\partial \dot{\phi}}{\partial J}\right)_{t}^{-1} \sqrt{\frac{2}{3}}
\end{aligned}
$$

where the subscript $t$ denotes that the derivatives are taken at the start of the increment. Throughout the paper $\rho=1$ will be used. It is noted, that $\operatorname{tr}(\mathbf{D})=\operatorname{tr}\left(\mathbf{D}^{\mathrm{e}}\right) \simeq 0$ due to plastic incompressibility where $\operatorname{tr}\left(\mathbf{D}^{p}\right) \equiv 0$.

\section{Computational Method}

An updated Lagrangian formulation [29, 30] based on the principle of virtual work is used. Disregarding body forces the incremental form of the principle of virtual work in terms of the Kirchhoff stress, $\tau_{i j}=\tau_{j i}$, is [31]

$$
\begin{array}{r}
\Delta t \int_{V}\left(\stackrel{\nabla}{\tau}_{i j} \delta D_{i j}-\sigma_{i j}\left(2 D_{i k} \delta D_{k j}-L_{k j} \delta L_{k i}\right)\right) \mathrm{d} V= \\
\Delta t \int_{S} \dot{T}_{i} \delta \dot{u}_{i} \mathrm{~d} S-\left[\int_{V} \sigma_{i j} \delta D_{i j} \mathrm{~d} V-\int_{S} T_{i} \delta \dot{u}_{i} \mathrm{~d} S\right]
\end{array}
$$

where $V$ is the volume and $S$ is the surface, $T_{i}=\sigma_{i j} n_{j}$ are the tractions and $\delta \dot{u}_{i}$ are the virtual velocities, all referred to the current deformed configuration. Therefore, $\tau_{i j}$ is identical to $\sigma_{i j}$. From the principle of virtual work the symmetric element stiffness matrix as well as the consistent nodal load vectors are established. The bracketed terms in Eq. (12) vanish if the current state satisfies equilibrium. However, due to numerical errors the solution tends to drift away from the true equilibrium path, and including the bracketed terms in Eq. (12) as an additional load term, prevents such drifting.

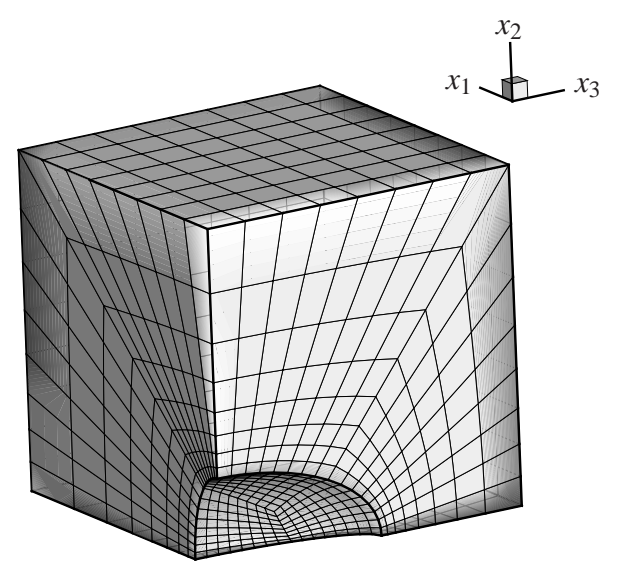

Fig. 2. Example of mesh used for evenly distributed ellipsoidal voids $\left(w_{1}=2\right.$ and $w_{3}=\frac{1}{2}$ as well as $L_{2} / L_{1}=L_{2} / L_{3}=1$ for an initial void volume fraction of $f_{0}=0.01$ ) using 1536 elements. 
For the numerical finite element solution the cell is discretisized using iso-parametric, quadratic 20-node brick elements with three translational degrees of freedom per node. Reduced $2 \times 2 \times 2$ Gauss integration is adopted. Fig. 2 shows an example of a mesh consisting of 1536 elements (22083 DOFs) for a cell given by $w_{1}=2$ and $w_{3}=\frac{1}{2}$ as well as $L_{2} / L_{1}=L_{2} / L_{3}=1$ with the void volume fraction $f_{0}=0.01$. In cases with $L_{2} / L_{1} \neq L_{2} / L_{3} \neq 1$, more elements are used. The elements are stretched, such that the elements are smaller near the void. In particular, as the primary load is in the $x_{2}$ direction, the elements for $x_{2}=0$ are rather thin in order to withstand the large deformation in the $x_{2}$ direction. The mesh shown in Fig. 2 has proven to be sufficiently fine, in order to obtain converged results. The finite element solution is obtained by parallelization of the system of equation using up to $20 \mathrm{CPU}$ 's. In each increment, the time step, $\Delta t$, for the next increment is adjusted according to $\left(\dot{\varepsilon}^{\mathrm{p}}\right)_{\max } \cdot \Delta t \leq 10^{-5}$, where $\left(\dot{\varepsilon}^{\mathrm{p}}\right)_{\text {max }}$ is the maximum effective plastic strain rate in any Gauss point.

\section{Numerical Results}

The effect of hydrostatic tension on void growth is very well understood from many previous investigations. Higher stress triaxiality gives much more rapid void growth, and this is also true for anisotropic plasticity. But such effects are not the aim of the present study, and therefore it has been chosen to consider only one stress triaxiality, $T=2$, in all the analyses here. Also, it is known that low triaxiality will tend to develop long narrow voids under axisymmetric tension (e.g. see Budiansky et al. [32]), or voids that close up in microcracks under simple shear (Tvergaard [33]), but again such effects are not the aim of the present study, and such effects of stress state are not illustrated under the rather high stress triaxiality applied here. Larger voids will tend to give earlier failure by void coalescence than small voids, but this will not be studied here where it has been chosen to consider only one initial void volume fraction, $f_{0}=0.01$ for various geometrical configurations defined by $w_{1}, w_{3}, L_{2} / L_{1}, L_{2} / L_{3}$. Finally, while the coefficients in (9) are varied, to consider different plastic anisotropies, only one set of the material parameters $\sigma_{0} / E, n$ and $m$ are considered.

Analyses are carried out for $\sigma_{0} / E=0.002, \mathrm{v}=$ $1 / 3, \dot{\varepsilon}_{0}=0.001 \mathrm{~s}^{-1}, \mathrm{~m}=0.01$ and $n=0.1$. The initial time step is $0.002 \mathrm{~s}$. Two of the plastic anisotropies studied in [21] are also considered here. They are both defined by $H=1.00$ and $L=M=N=9.60$ in Eq. (9), corresponding to the experiments by Moen et al. [34] on aluminum alloy Al-7108-T7. Furthermore, $F=0.40$ and $G=7.33$ for the anisotropy labelled II, while $F=2.50$ and $G=0.30$ for the anisotropy labelled IV. These values of $F$ and $G$ are slightly more extreme compared to the experiments by Moen et al. [34], which gave $F=0.70$ and $G=3.33$. Isotropic results will also be presented, i.e. for $F=G=H=1$ and $L=M=N=3.00$. Fig. 3 illustrates the corresponding initial yield surfaces in the $\left(\sigma_{11}, \sigma_{22}\right)$-plane for $\sigma_{33}=\sigma_{12}=\sigma_{13}=\sigma_{23}=0$. Comparing to the isotropic Mises yield surface, it is seen, that while anisotropy II expands the elastic region anisotropy IV shrinks it.

True stress versus logarithmic strain curves for the $x_{2}$ direction will be shown. These quantities are calculated as

$$
\begin{gathered}
\Sigma_{2}=\int_{S} T_{2} \mathrm{~d} S=\int_{0}^{L_{1}+\Delta L_{1}} \int_{0}^{L_{3}+\Delta L_{3}}\left[T_{2}\right]_{L_{2}+\Delta L_{2}} \mathrm{~d} x_{1} \mathrm{~d} x_{3} \\
E_{2}=\ln \left(1+\frac{\Delta L_{2}}{L_{2}}\right)
\end{gathered}
$$

where $S$ is the surface in the current deformed geometry, whereas $\Delta L_{1}, \Delta L_{2}$ and $\Delta L_{3}$ are the accumulated elongations of the three loaded cell sides. The current void volume fraction, $f$, will also be shown. It will be found by numerical integration of the void volume divided by the current volume of the cell.

The first cases analysed consider a material with isotropic plasticity to be able to make a comparison with the results to be shown subsequently for anisotropy. Fig. 4 shows the true stress versus the true strain in the $x_{2}$ direction, and the current void volume fraction $f$ versus the true strain in cases where the unit cell is initially a cube, so that the void spacings in the three coordinate directions are initially equal. The stress triaxiality $T=2$ is here obtained by taking equal stresses in the two transverse directions, with $\kappa_{1}=\kappa_{3}=5 / 8$. The curves for $w_{1}=w_{3}<1$ represent oblate voids, while the curves for $w_{1}=w_{3}>1$ represent prolate voids, and curves for an initially spherical void, $w_{1}=w_{3}=1$, are included for

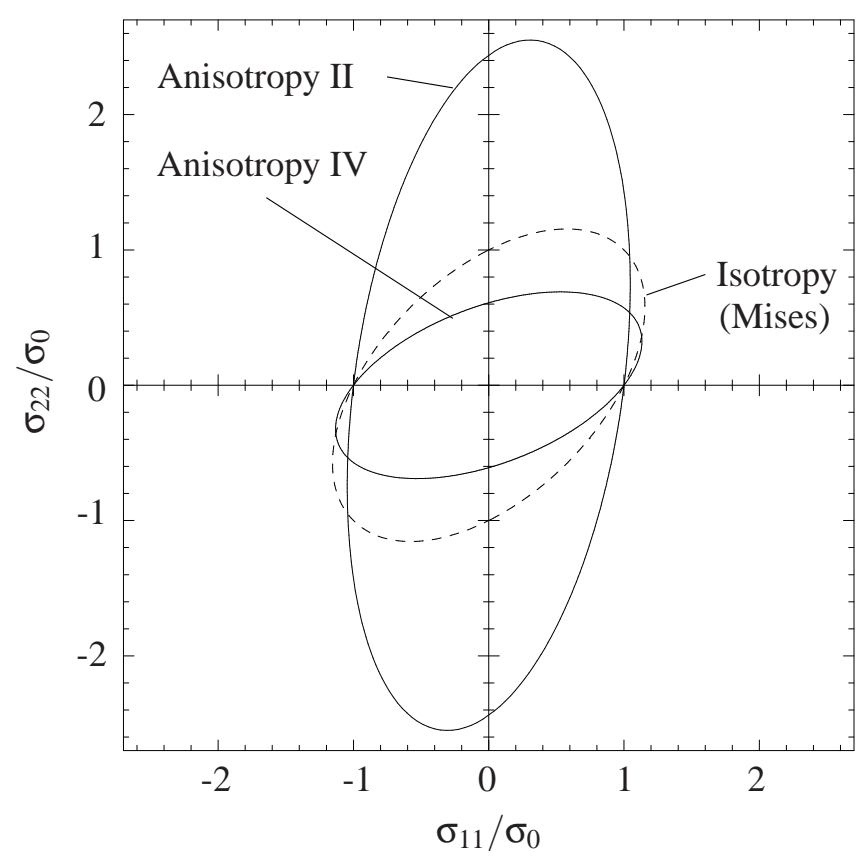

Fig. 3. Initial yield surfaces shown in the $\left(\sigma_{11}, \sigma_{22}\right)$-plane for $\sigma_{33}=\sigma_{12}=\sigma_{13}=\sigma_{23}=0$. Two different anisotropic Hill yield surfaces are shown: (II) $F=0.40$ and $G=7.33$ (IV) $F=2.50$ and $G=0.30$, while both have $H=1.00, N=L=M=9.60$ in Eq. (9). The isotropic Mises yield surface is also shown. 

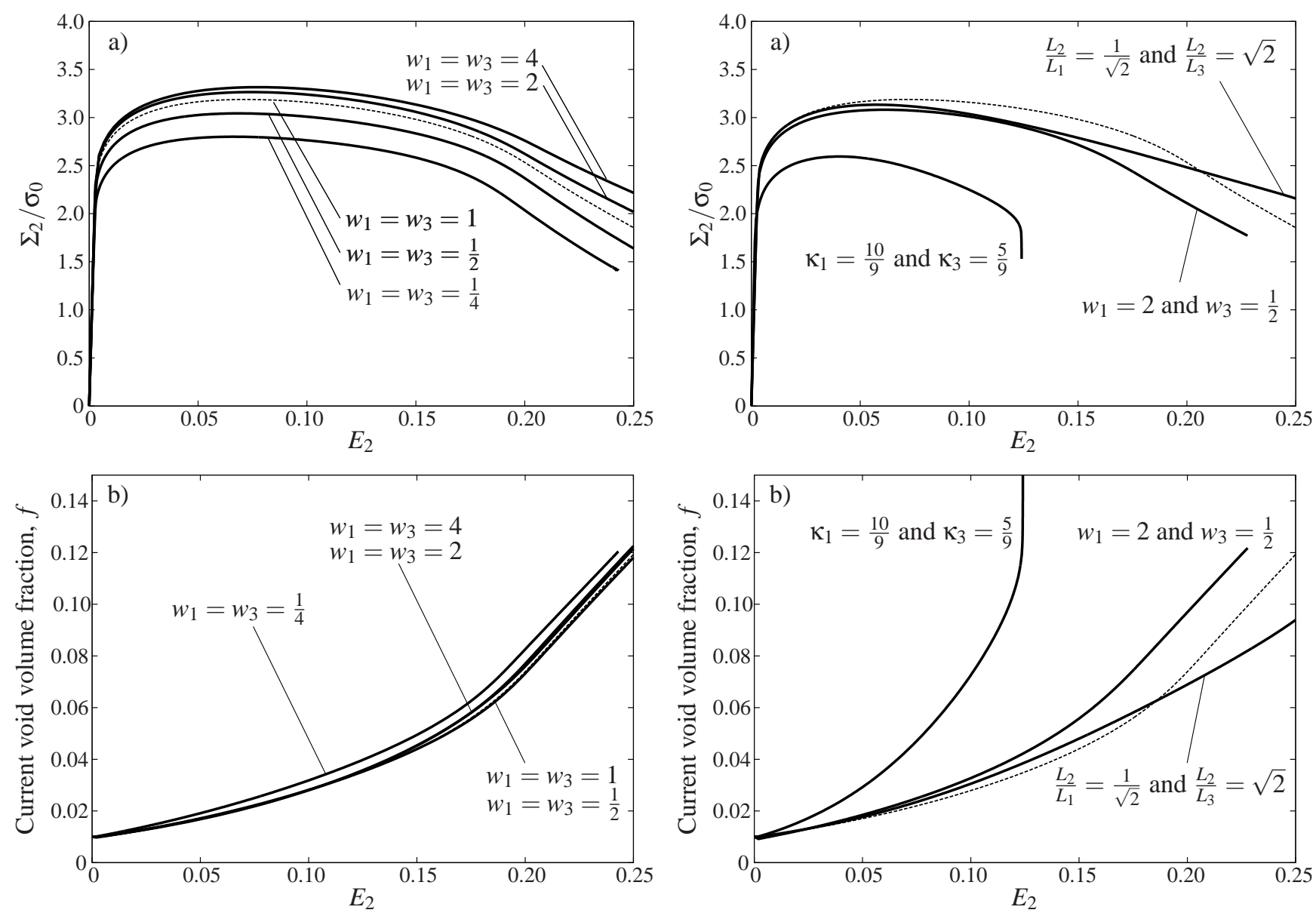

Fig. 4. Effects of void shape, $w_{1}$ and $w_{3}$, for isotropic plasticity, equally distributed voids, $L_{2} / L_{1}=L_{2} / L_{3}=1$, and $\kappa_{1}=\kappa_{3}=\frac{5}{8}$ $(T=2)$. a) Normalized stress-strain response in the primary load direction. b) Void volume fraction evolution.

reference. It is seen that the oblate voids reduce the stress level, while the prolate voids allow for a higher stress level during the tensile loading. The same tendency was found by Pardoen and Hutchinson [35] in axisymmetric cell model studies and Cao et al. [36] for a 3D cell. The evolution of the void volume fraction during the tensile loading differs rather little between the five cases. Subsequently, the curve for isotropic plasticity with $w_{1}=w_{3}=L_{2} / L_{1}=L_{2} / L_{3}=1$ and $\kappa_{1}=\kappa_{3}=5 / 8$ will be used as a reference curve to illustrate the influence of various parameters. This reference curve will be shown by a dashed line in the following figures.

Also in Fig. 5 the material follows isotropic plasticity, but here other parameters are varied, and comparison is made with the reference case shown by the dashed curves, which was also part of Fig. 4. When $L_{2} / L_{1}=1 / \sqrt{2}$ and $L_{2} / L_{3}=\sqrt{2}$ the spacing between the spherical voids is initially larger in the $x_{1}$ direction than in the $x_{2}$ direction, and smaller in the $x_{3}$ direction. In this case the stress level is initially lower than the reference case, but then decays more slowly, and the void volume fraction grows less rapidly after the initial stage. At the curves for $w_{1}=2$ and $w_{3}=1 / 2$ the voids have initially a general ellipsoidal shape, with different

Fig. 5. Effects of void shape, $w_{1}$ and $w_{3}$, void spacing, $L_{2} / L_{1}$ and $L_{2} / L_{3}$, and stress ratio, $\kappa_{1}$ and $\kappa_{3}$ for isotropic plasticity. When nothing else is stated the curves have $w_{1}=w_{3}=1, L_{2} / L_{1}=$ $L_{2} / L_{3}=1$ and $\kappa_{1}=\kappa_{3}=\frac{5}{8}(T=2)$. a) Normalized stress-strain response in the primary load direction. b) Void volume fraction evolution.

lengths of the three half axes, see Fig. 1. These curves show lower stress levels and more rapid void growth than found for the reference case. Finally, $\kappa_{1}=10 / 9$ and $\kappa_{3}=5 / 9$ represents a case where the principal stress $\Sigma_{1}$ is larger than $\Sigma_{2}$, while $\Sigma_{3}$ is lower. In this case the stress level of $\Sigma_{2}$ remains significantly lower than the other curves, and the void volume fraction grows much faster as a function of $E_{2}$. This is related to the fact that the larger stress component in the $x_{1}$ direction results in a more rapid growth of $E_{1}$ than $E_{2}$, such that $E_{1}=0.327$ at the end of the curve, $E_{2}=0.124$.

Fig. 6 illustrates the effect of plastic anisotropies in cases where the voids are initially axisymmetric, $w_{1}=w_{3}$, uniformly spaced in all three coordinate directions, $L_{2} / L_{1}=$ $L_{2} / L_{3}$, and with equal stresses in the two transverse directions, $\kappa_{1}=\kappa_{3}$. The anisotropies labelled II or IV are specified in Fig. 3. It is seen that generally anisotropy II results in much higher stress peaks and much more rapid void growth than found for the reference case, and anisotropy IV results in lower stress peaks, decaying slowly, and in less rapid void growth. This agrees well with anisotropy II having a larger 

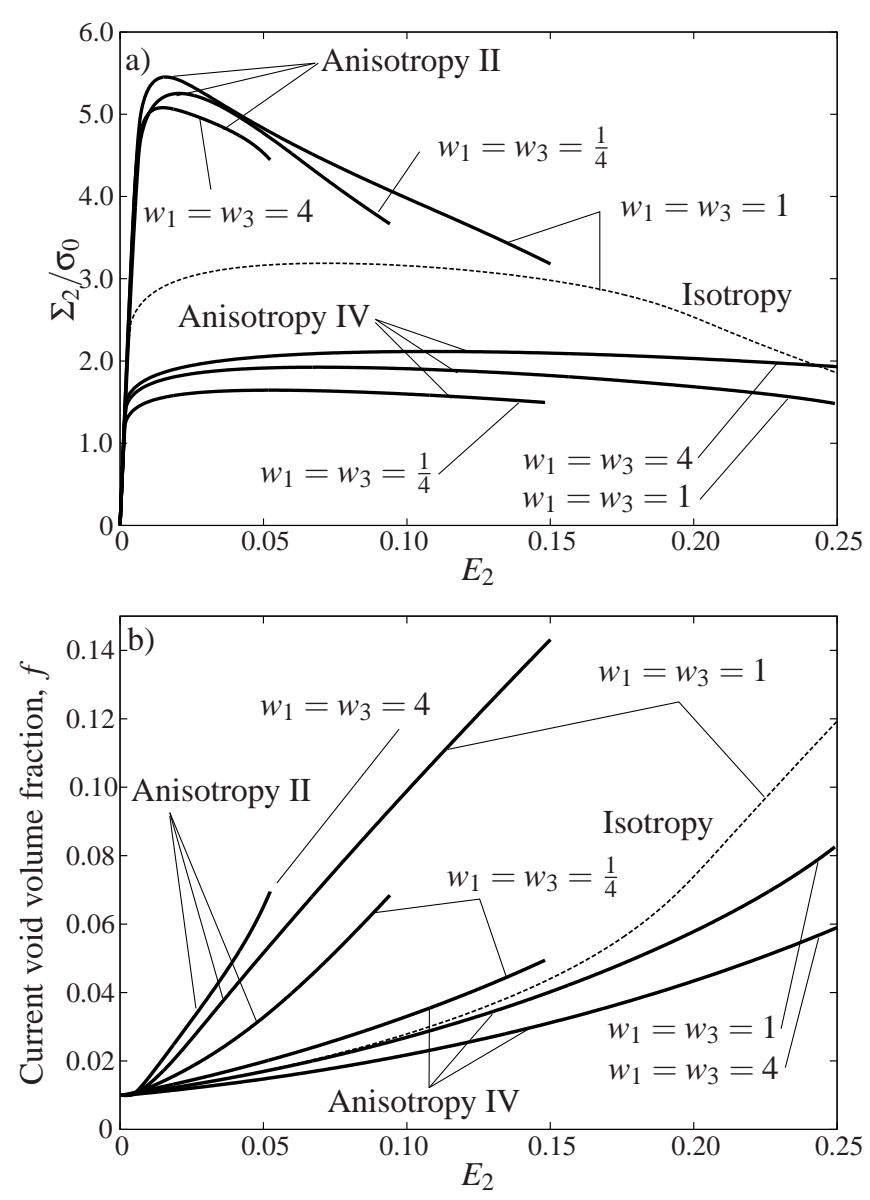

Fig. 6. Effects of axi-symmetric void shape, $w_{1}=w_{3}$, for anisotropic plasticity and equally distributed voids, $L_{2} / L_{1}=$ $L_{2} / L_{3}=1, \kappa_{1}=\kappa_{3}=\frac{5}{8}(T=2)$. a) Stress-strain response in the primary load direction. b) Void volume fraction evolution.

yield stress in the $x_{2}$ direction compared to anisotropy IV, Fig. 3. In Fig. 4, for an isotropic matrix, it was seen that oblate voids, $w_{1}=w_{3}<1$, reduce the stress level, whereas the stresses are higher for prolate voids. This is also seen in Fig. 6 for anisotropy IV, but for anisotropy II the effect is opposite. It is also noted, that case of spherical voids in Fig. 4 falls in between the oblate and prolate cases, which is only the case for anisotropy IV in Fig. 6. In Fig. 4 there was not much difference between the rate of void growth for the different cases considered, but in Fig. 6 there is clear difference between the void volume fractions for prolate or oblate voids, such that the oblate voids grow faster in the case of anisotropy IV but slower in the case of anisotropy II. Fig. 7 shows the contours of the effective plastic strain, $\varepsilon^{p}$, at the end of the curve in Fig. 6 for $w_{1}=w_{3}=\frac{1}{4}$ with anisotropy IV. The initial cell is also indicated. Even though a symmetric geometry as well as symmetric loading are considered, a high local plastic strain develops at the surface of the void near the $x_{1}$-axis, which is caused by the plastic anisotropy. The macroscopic compressive strain in the $x_{1}$ direction is approximately half the strain in the $x_{3}$ direction, i.e. $E_{1}=-0.031$ and $E_{3}=-0.073$, respectively. This is opposite to the case with anisotropy II, where the macroscopic compressive strain

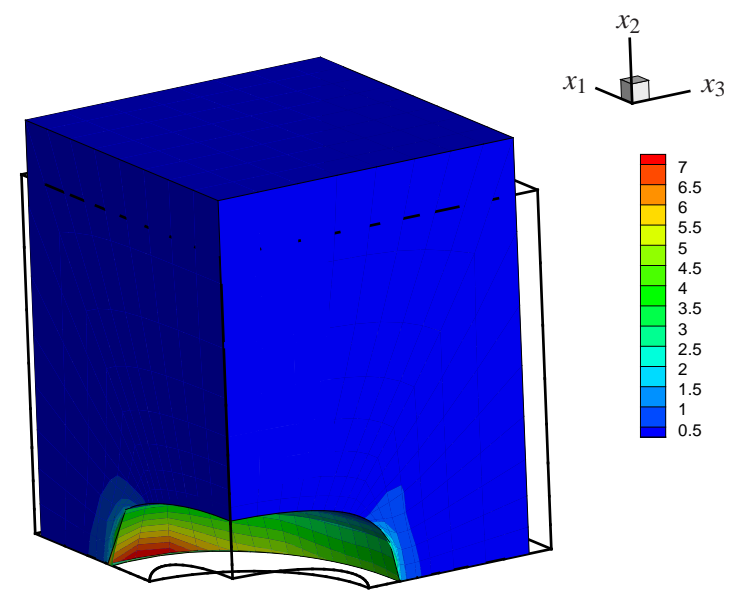

Fig. 7. Contours of effective plastic strain, $\varepsilon^{\mathrm{p}}$, at an overall strain, $E_{2}=0.147$, in Fig. 6, for $w_{1}=w_{3}=\frac{1}{4}, L_{2} / L_{1}=L_{2} / L_{3}=1$, $\kappa_{1}=\kappa_{3}=\frac{5}{8}(T=2)$ with plastic anisotropy IV (color print available online).

in the $x_{1}$ direction is approximately twice the strain in the $x_{3}$ direction, i.e. $E_{1}=-0.018$ and $E_{3}=-0.009$, respectively. However, the strain reached is significantly smaller.

For a general ellipsoidal void, specified by $w_{1}=2$ and $w_{3}=1 / 2$, Fig. 8 gives a comparison of the effect of the two different anisotropies. For an isotropic matrix material Fig. 5 has shown that this void shape gives a behaviour a little different from the reference curve, but Fig. 8 shows that the difference is much larger when the void is embedded in one of the anisotropic materials. In the case of anisotropy II the peak stress is much higher than that on the reference curve and also the corresponding growth of the void volume fraction is much faster, whereas the case of anisotropy IV shows a lower stress peak and a slower growth of the void volume fraction. In fact, for both anisotropies the curves are in the range of the curves found in Fig. 6 for the same anisotropies, with axisymmetric voids. The curves in Fig. 8 are very close to those in Fig. 6 for $w_{1}=w_{3}=1$.

Fig. 9 illustrates results of the two different anisotropies for a case where the spacing between the spherical voids is initially larger in the $x_{1}$ direction than in the $x_{2}$ direction, and smaller in the $x_{3}$ direction, and for a case where the principal stress $\Sigma_{1}$ is larger than $\Sigma_{2}$, while $\Sigma_{3}$ is lower. In both cases the corresponding predictions for an isotropic matrix are shown in Fig. 5. In the case of different spacings, $L_{2} / L_{1}=1 / \sqrt{2}$ and $L_{2} / L_{3}=\sqrt{2}$, the curves are rather close to those shown in Fig. 8 for the two anisotropies, although there are small quantitative differences. The case of different principal stresses in the three coordinate directions, $\kappa_{1}=10 / 9$ and $\kappa_{3}=5 / 9$, resulted in rather unusual behaviour in Fig. 5, with the curves ending by a steep load drop and a steep increase of the void volume fraction. In Fig. 9 for anisotropy II the stress reaches a value well above that in Fig. 5, but the curves end at a rather small strain, 

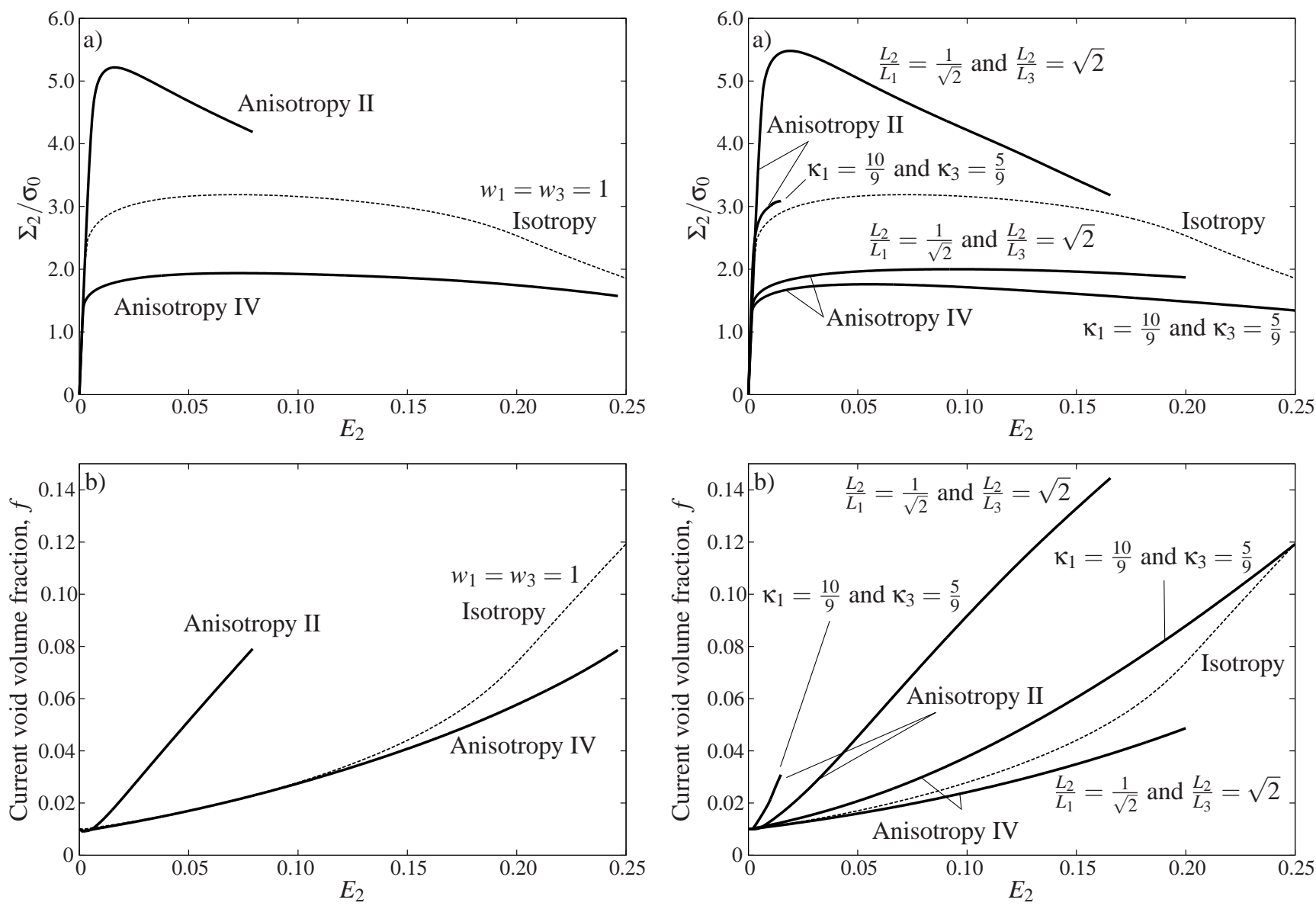

Fig. 8. Effects of general void shape, $w_{1}=2$ and $w_{3}=\frac{1}{2}$, for anisotropic plasticity and equally distributed voids, $L_{2} / L_{1}=$ $L_{2} / L_{3}=1, \kappa_{1}=\kappa_{3}=\frac{5}{8}(T=2)$. a) Stress-strain response in the primary load direction. b) Void volume fraction evolution.

$E_{2}=0.015$, due to a numerical instability where very large strains at a point on the void surface near the $x_{2}$-axis result in strong mesh distortion. The deformed unit cell and contours of effective plastic strain at the end point of the curve are shown in Fig. 10, with the shape of the initial cell indicated. In this case the largest principal stress is in the $x_{1}$ direction, and for anisotropy II the deformed cell in Fig.10 shows that the strain in this direction has grown much larger, $E_{1}=0.396$. With the very small growth of $E_{2}$ indicated by Fig. 10 there is the possibility that $E_{2}$ would start to decay during the continued void growth, but this has been tested using the finite element-Rayleigh Ritz method, and it has been found that a change of sign for $E_{2}$ is not the reason for the numerical instability. For anisotropy IV in Fig. 9 the case of different principal stresses in the three coordinate directions gives a rather flat stress-strain curve with corresponding slow growth of the void volume fraction.

In Fig. 11 two deviations from the standard unit cell are considered simultaneously, the voids have the general ellipsoidal shape also considered in some cases of Figs. 5 and $8, w_{1}=2$ and $w_{3}=1 / 2$, and the principal stress $\Sigma_{1}$ is larger than $\Sigma_{2}$, while $\Sigma_{3}$ is lower, as also considered in some cases of Figs. 5 and 9. For anisotropy II the curves in Fig.

Fig. 9. Effects of spacing, $L_{2} / L_{1}$ and $L_{2} / L_{3}$, and stress ratio, $\kappa_{1}$ and $\kappa_{3}$, for anisotropic plasticity with spherical voids, $w_{1}=w_{3}=1$. When nothing else is stated, the curves have $L_{2} / L_{1}=L_{2} / L_{3}=1$ and $\kappa_{1}=\kappa_{3}=\frac{5}{8}(T=2)$. a) Stress-strain response in the primary load direction. b) Void volume fraction evolution.

11 show some similarity with the curves for $\kappa_{1}=10 / 9$ and $\kappa_{3}=5 / 9$, Fig. 9, in that a rather low value of the strain is reached, $E_{2}=0.027$, and as was described in relation to Fig. 9 the value of the strain in the $x_{1}$ direction has grown much larger, $E_{1}=0.778$. For anisotropy IV the stress-strain curve is rather flat with a low peak as in the corresponding case in Fig. 9, but the void volume fraction grows more rapidly in Fig. 11. It may be concluded that the rather drastic difference between the two anisotropies found in Fig. 9 for $\kappa_{1}=10 / 9$ and $\kappa_{3}=5 / 9$ with initially spherical voids is less drastic in Fig. 11 with the general ellipsoidal shape of the voids, but the trends are still the same.

\section{Discussion}

The present 3D cell model analyses of void growth show that there is a clear interaction between the different effects considered, the plastic anisotropies, the void shape, the different void spacings in different coordinate directions, and the differences in the principal macroscopic stresses. The interactions between these four types of effects are the aim of the study. Therefore, other parameters are not varied, and 


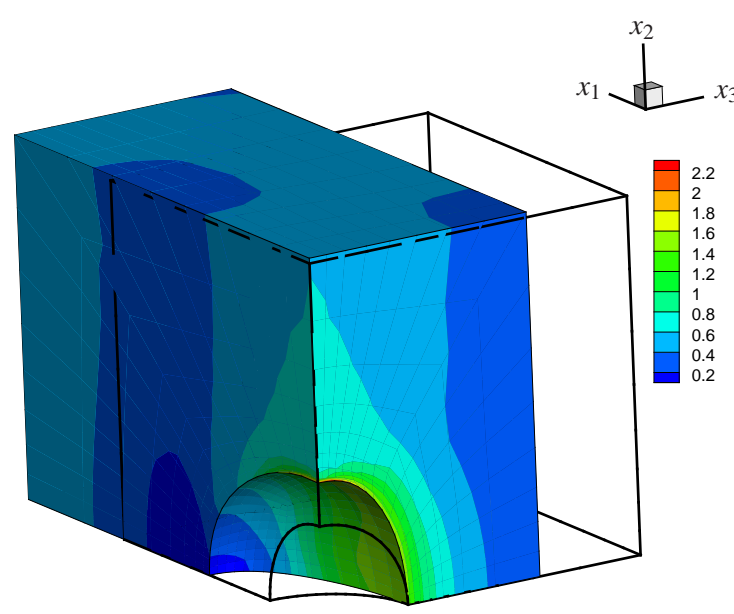

Fig. 10. Contours of effective plastic strain, $\varepsilon^{\mathrm{p}}$, at an overall strain, $E_{2}=0.015$, in Fig. 9, for $w_{1}=w_{3}=1, L_{2} / L_{1}=L_{2} / L_{3}=1$, $\kappa_{1}=\frac{10}{9}$ and $\kappa_{3}=\frac{5}{9}(T=2)$ with plastic anisotropy II (color print available online).

only one stress triaxiality, one initial void volume fraction, and one set of reference values for the uniaxial stress-strain curve are considered.

The first studies, for isotropic plasticity, show that each of the three other effects considered, the void shape, the void spacings, and deviations from equal transverse stresses, have an effect on the overall stress-strain curve and on the rate of growth of the void volume fraction. For axisymmetric voids, oblate shapes give a reduced stress level, whereas prolate shapes allow for higher stresses. The general ellipsoidal void shapes considered result in a reduced stress level.

When plastic anisotropy is added to the three other effects, the differences are much more visible. First of all, one of the plastic anisotropies considered gives much higher stress peaks than the other anisotropy. But there is also an interaction between the plastic anisotropies and the void shapes, such that the difference between prolate and oblate voids in the anisotropic plasticity matrices is larger than was found for isotropic plasticity. For one of the anisotropies considered the oblate voids result in a lower stress peak than the prolate voids, as was found for isotropic plasticity, but the other anisotropy gives the opposite result that the oblate voids results in the highest stress peak. The general ellipsoidal void shapes considered result in stress-strain curves and void volume growth curves rather close to those found for spherical voids with the same plastic anisotropy. The studies carried out for different spacings of the voids in the three coordinate directions did not show much difference from predictions for uniform spacings.

Considering differences in the transverse macroscopic principal stresses it has been found that this can strongly affect the material response, in particular when combined with plastic anisotropy. And the response is very different for the different plastic anisotropies considered here. For anisotropy
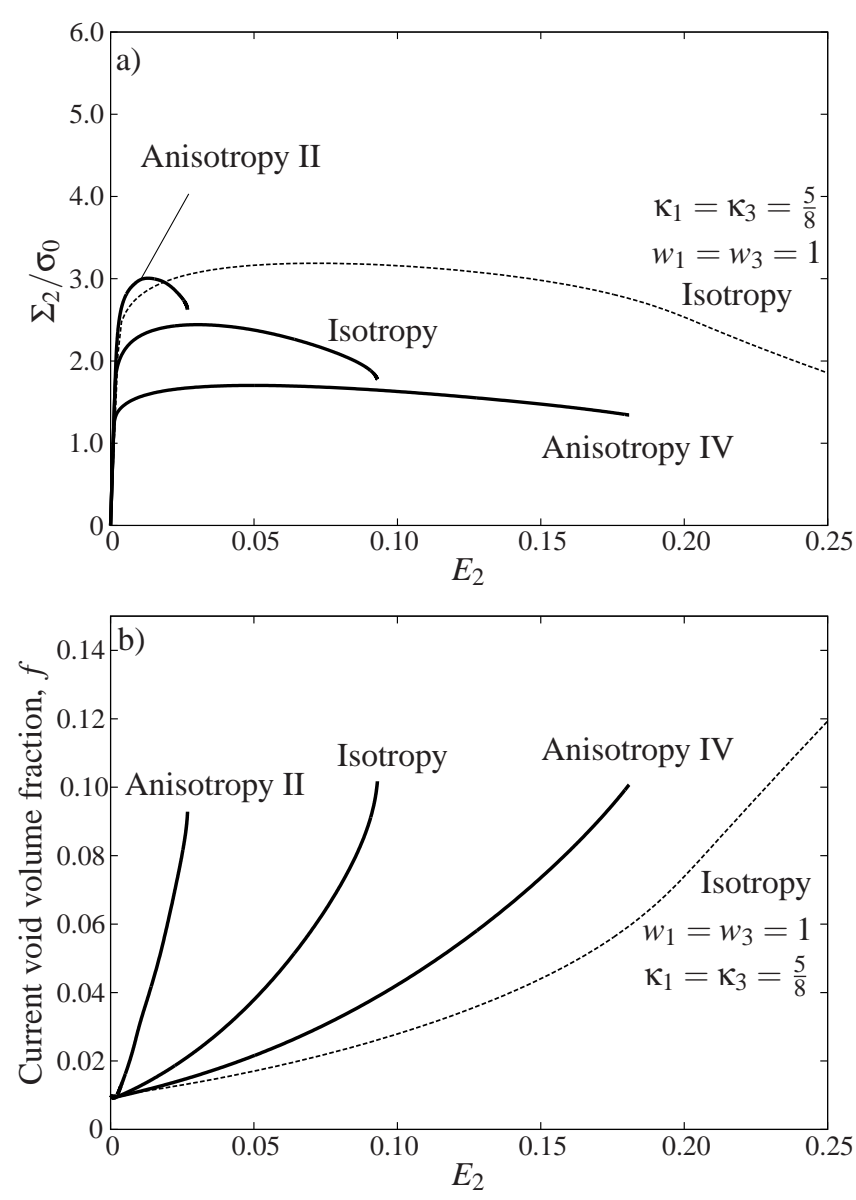

Fig. 11. Plasticity effects for equally distributed voids, $L_{2} / L_{1}=$ $L_{2} / L_{3}=1$, of general shape, $w_{1}=2$ and $w_{3}=\frac{1}{2}$ with the stress ratios $\kappa_{1}=\frac{10}{9}$ and $\kappa_{3}=\frac{5}{9}(T=2)$. a) Stress-strain response in the primary load direction. b) Void volume fraction evolution.

II it leads to a very large strain in the transverse direction where the stress is largest, while for anisotropy IV the largest strain is in the primary load direction. When the different transverse macroscopic stresses and plastic anisotropy are also combined with initially ellipsoidal voids these three effects interact further, still with a drastic difference between the two plastic anisotropies considered.

A limitation of the cell studies performed here is that the orientation of plastic anisotropy initially has to be aligned with the cell sides in order not to violate the symmetry conditions. Also, only normal loads can be applied and the principal axes of the ellipsoidal void have to be aligned with the cell sides as well. In order to allow for general orientations of anisotropy and voids as well as shear loads, a larger cell with periodic boundary conditions is needed [37]. In the most general case the full void (eight times as large cell) has to be used. However, in the special case, where the principal axes of anisotropy or the principal axes of the void are inclined only relative to one of the cell sides, a cell containing half the void will be sufficient (four times as large cell). 


\section{References}

[1] McClintock, F. A., 1968. "A criterion for ductile fracture by growth of holes". Journal of Applied Mechanics, 35(2), pp. 363-371.

[2] Rice, J. R., and Tracey, D. M., 1969. "On the ductile enlargement of voids in triaxial stress fields". Journal of the Mechanics and Physics of Solids, 17, pp. 201217.

[3] Garrison Jr, W. M., and Moody, N. R., 1987. "Ductile fracture". Journal of Physics and Chemistry of Solids, 48(11), pp. 1035-1074.

[4] Tvergaard, V., 1990. "Material failure by void growth to coalescence". Advances in Applied Mechanics, 27, pp. 83-151.

[5] Benzerga, A., A., and Leblond, J.-B., 2010. "Ductile fracture by void growth to coalescence". Advances in Applied Mechanics, 44, pp. 169-305.

[6] Benzerga, A. A., Leblond, J. B., Needleman, A., and Tvergaard, V., 2016. "Ductile failure modeling". International Journal of Fracture, 201(1), pp. 29-80.

[7] Gurson, A., L., 1977. "Continuum theory of ductile rupture by void nucleation and growth - I. Yield criteria and flow rules for porous ductile media". Journal of Engineering Materials and Technology-transactions of the Asme, 99(1), pp. 2-15.

[8] Tvergaard, V., 1981. "Influence of voids on shear band instabilities under plane strain conditions". International Journal of Fracture, 17(4), pp. 389-407.

[9] Tvergaard, V., and Needleman, A., 1984. "Analysis of the cup-cone fracture in a round tensile bar". Acta Metallurgica, 32(1), pp. 157-169.

[10] Hill, R., 1948. "A theory of the yielding and plastic flow of anisotropic metals". Proceedings of the Royal Society of London, A193, pp. 281-297.

[11] Hill, R., 1950. The Mathematical Theory of Plasticity. The Clarendon Press, Oxford.

[12] Benzerga, A., A., and Besson, J., 2001. "Plastic potentials for anisotropic porous solids". European Journal of Mechanics A-solids, 20(3), pp. 397-434.

[13] Keralavarma, S. M., and Benzerga, A. A., 2010. "A constitutive model for plastically anisotropic solids with non-spherical voids". Journal of the Mechanics and Physics of Solids, 58(6), pp. 874-901.

[14] Morin, L., Madou, K., Leblond, J.-B., and Kondo, D., 2014. "A new technique for finite element limitanalysis of Hill materials, with an application to the assessment of criteria for anisotropic plastic porous solids". International Journal of Engineering Science, 74, pp. 65-79.

[15] Steglich, D., Wafai, H., and Besson, J., 2010. "Interaction between anisotropic plastic deformation and damage evolution in Al 2198 sheet metal". Engineering Fracture Mechanics, 77(17), pp. 3501-3518.

[16] Chien, W., Y., Pan, J., and Tang, S., C., 2001. "Modified anisotropic Gurson yield criterion for porous ductile sheet metals". Journal of Engineering Materials and Technology-transactions of the Asme, 123(4), pp. 409-416.
[17] Wang, D.-A., Pan, J., and Liu, S.-D., 2004. “An anisotropic Gurson yield criterion for porous ductile sheet metals with planar anisotropy". International Journal of Damage Mechanics, 13, pp. 7-33.

[18] Dæhli, L. E. B., Faleskog, J., Børvik, T., and Hopperstad, O. S., 2017. "Unit cell simulations and porous plasticity modelling for strongly anisotropic FCC metals". European Journal of Mechanics A-solids, 65, pp. 360-383.

[19] Barlat, F., Aretz, H., Yoon, J. W., Karabin, M. E., Brem, J. C., and Dick, R. E., 2005. "Linear transfomationbased anisotropic yield functions". International Journal of Plasticity, 21(5), pp. 1009-1039.

[20] Srivastava, A., and Needleman, A., 2015. "Effect of crystal orientation on porosity evolution in a creeping single crystal". Mechanics of Materials, 90, pp. 10-29.

[21] Legarth, B. N., and Tvergaard, V., 2010. "3D analyses of cavitation instabilities accounting for plastic anisotropy". Zeitschrift Fuer Angewandte Mathematik Und Mechanik, 90(9), pp. 701-709.

[22] Tvergaard, V., 1976. "Effect of thickness inhomogeneities in internally pressurized elastic-plastic spherical shells". Journal of the Mechanics and Physics of Solids, 24, pp. 291-304.

[23] Dafalias, Y. F., 1985. "A missing link in the macroscopic constitutive formulation of large plastic deformation". In Plasticity Today, Modelling, Methods and Applications, A. Sawczuk and G. Bianchi, eds., Elsevier, Amsterdam, pp. 135-151.

[24] Dafalias, Y. F., 1985. "The plastic spin”. Transactions of the ASME. Journal of Applied Mechanics, 52(4), pp. 865-871.

[25] Dafalias, Y. F., 1993. "On multiple spins and texture development. Case study: kinematic and orthotropic hardening". Acta Mechanica, 100, pp. 171-194.

[26] Legarth, B. N., 2008. "Necking of anisotropic microfilms with strain-gradient effects". Acta Mechanica Sinica, 24(5), pp. 557-567.

[27] Peirce, D., Shih, C. F., and Needleman, A., 1984. "A tangent modulus method for rate dependent solids". Computers and Structures, 18, pp. 875-887.

[28] Kuroda, M., and Tvergaard, V., 2001. "Plastic spin associated with a non-normality theory of plasticity". European Journal of Mechanics - A/Solids, 20(6), pp. 893-905.

[29] McMeeking, R. M., and Rice, J. R., 1975. "Finiteelement formulations for problems of large elasticplastic deformation". International Journal of Solids and Structures, 11, pp. 601-616.

[30] Yamada, Y., and Sasaki, M., 1995. "Elastic-plastic large deformation analysis program and lamina compression test". International Journal of Mechanics, 37(7), pp. 691-707.

[31] Legarth, B. N., 2007. "Strain-gradient effects in anisotropic materials". Modelling and Simulation in Materials Science and Engineering, 15, pp. S71-S81.

[32] Budiansky, B., Hutchinson, J. W., and Slutsky, S., 1982. "Void growth and collapse in viscous solids". 
Mechanics of Solids, The Rodney Hill $60^{\text {th }}$ Anniversary Volume, Eds. H.G. Hopkins and M.J. Sewell, 90, pp. 13-45.

[33] Tvergaard, V., 2009. "Behaviour of voids in a shear field". International Journal of Fracture, 158(1), pp. 41-49.

[34] Moen, L. A., Langseth, M., and Hopperstad, O., 1998. "Elastoplastic buckling of anisotropic aluminum, plate elements". Journal of Structural Engineering, pp. 712719.

[35] Pardoen, T., and Hutchinson, J. W., 2000. "An extended model for void growth and coalescence". Journal of the Mechanics and Physics of Solids, 48(12), pp. 24672512.

[36] Cao, T. S., Mazière, M., Danas, K., and Besson, J., 2015. "A model for ductile damage prediction at low stress triaxialities incorporating void shape change and void rotation". International Journal of Solids and Structures, 63, pp. 240-263.

[37] Legarth, B. N., 2004. "Unit cell debonding analyses for arbitrary orientations of plastic anisotropy". International Journal of Solids and Structures, 41(26), pp. 7267-7285. 